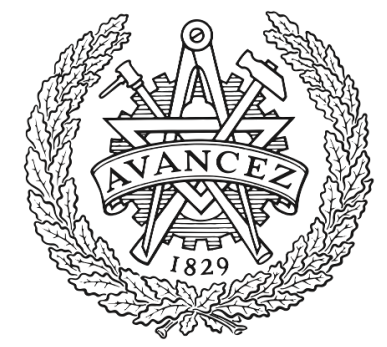

CHALMERS

UNIVERSITY OF TECHNOLOGY

\title{
Luminescence Quenching via Deep Defect States: A Recombination Pathway via Oxygen Vacancies in Ce-Doped YAG
}

Downloaded from: https://research.chalmers.se, 2023-04-26 03:28 UTC

Citation for the original published paper (version of record):

Linderälv, C., Åberg, D., Erhart, P. (2021). Luminescence Quenching via Deep Defect States: A Recombination Pathway via Oxygen Vacancies in Ce-Doped YAG. Chemistry of Materials, 33(1): 73-80.

http://dx.doi.org/10.1021/acs.chemmater.0c02449

N.B. When citing this work, cite the original published paper. 


\title{
Luminescence Quenching via Deep Defect States: A Recombination Pathway via Oxygen Vacancies in Ce-Doped YAG
}

\author{
Christopher Linderälv, Daniel Åberg, and Paul Erhart*
}

Cite This: Chem. Mater. 2021, 33, 73-80

Read Online

ABSTRACT: Luminescence quenching via nonradiative recombination channels limits the efficiency of optical materials such as phosphors and scintillators and therefore has implications for conversion efficiency and device lifetimes. In materials such as Cedoped yttrium aluminum garnet (YAG:Ce), quenching shows strong dependence on both temperature and activator concentration, limiting the fabrication of high-intensity white-light emitting diodes with high operating temperatures. Here, we reveal by means of firstprinciples calculations an efficient recombination mechanism in YAG:Ce that involves oxygen vacancies and gives rise to thermally activated concentration quenching. We demonstrate that the key requirements for this mechanism to be active are localized states with strong electron-phonon coupling. These conditions are commonly found for intrinsic defects such as anion vacancies in wide band gap materials. The present findings are therefore relevant to a broad class of optical materials and shine light on thermal quenching mechanisms in general.

\section{INTRODUCTION}

Optical materials with wide band gaps, such as phosphors and scintillators, enable the conversion of photons and high-energy radiation into one or several photons of lower energy. This allows for applications in, for example, lighting (phosphors), radiation detection (scintillators), and lasing. The performance of these materials is limited by nonradiative decay processes, most commonly via phonons and/or defects, which compete with the conversion into photons. Luminescence becomes, in particular, quenched at higher temperatures, which imposes limits, for example, with regard to the maximum operating power or the operation temperature. Despite extensive research, the mechanisms responsible for luminescence quenching are still not fully understood. ${ }^{1,2}$

Cerium-doped yttrium aluminum garnet (YAG:Ce, $\left.\mathrm{Y}_{3-x} \mathrm{Ce}_{x} \mathrm{Al}_{5} \mathrm{O}_{12}\right)$ is a yellow phosphor that is widely employed in rare earth-based solid-state lightning. ${ }^{3,4}$ The optical transitions that are exploited in solid-state lightning occur between $4 \mathrm{f}^{1} 5 \mathrm{~d}$ and $4 \mathrm{f}^{0} 5 \mathrm{~d}^{1}$ states of Ce. Several mechanisms have been suggested to explain quenching in this material ${ }^{5,6}$ (Figure 1):

(i) In principle, it could be possible for the system to undergo a thermally activated landscape crossover via multiphonon processes between the ground (Ce: $4 \mathrm{f}^{1} 5 \mathrm{~d}^{0}$ ) and excited states (Ce: $\left.4 \mathrm{f}^{0} 5 \mathrm{~d}^{1}\right){ }^{7,8}$ In this regard, it has been observed that the Debye temperature increases with $\mathrm{Ce}$ concentration, resulting in a larger high-frequency phonon population at lower temperatures, which in turn enhances multiphonon processes. ${ }^{9}$ Earlier studies, however, concluded that emission of high-frequency phonons could not explain the temperature dependence of the nonradiative decay in YAG:Ce. ${ }^{10}$

(ii) While the onset temperature for luminescence quenching is above $600 \mathrm{~K}$ for Ce concentrations below $1 \%$, it drops to around $400 \mathrm{~K}$ if the Ce content reaches the percent level. ${ }^{1}$ Based on this observation, the reduction in luminescence has been attributed to thermally activated concentration quenching, in which the excitation energy is resonantly transferred between Ce atoms until it is eventually dissipated at a, not further specified, killer center.

(iii) Finally, according to the thermal ionization model, an electron is emitted from the excited state of Ce to the conduction band edge $\left(4 \mathrm{f}^{0} 5 \mathrm{~d}^{1} \rightarrow 4 \mathrm{f}^{0} 5 \mathrm{~d}^{0}+e^{\prime}\right)$ or a defect level. The rate of this process has been shown to be

Received: June 11, 2020

Revised: December 5, 2020

Published: December 22, 2020 

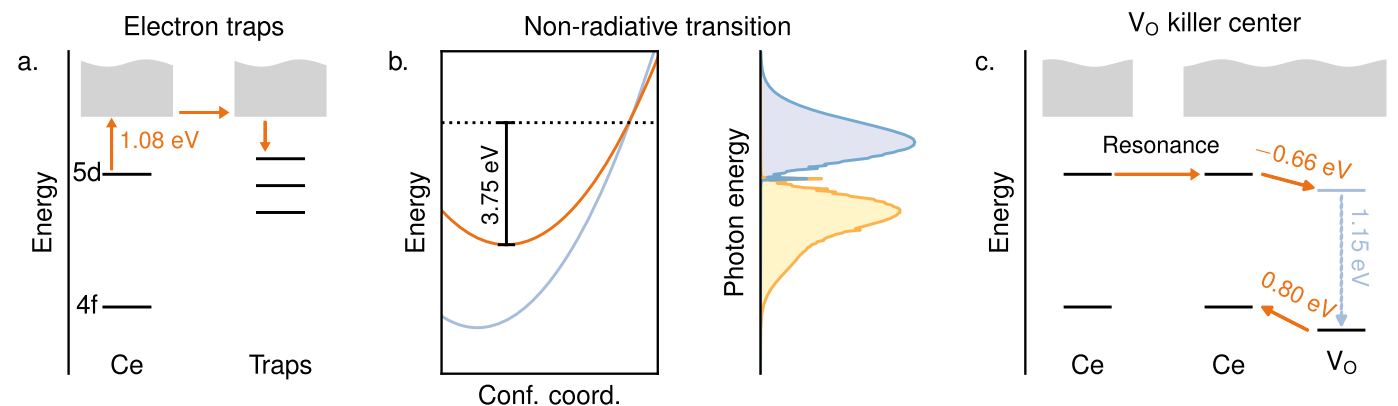

Figure 1. Schematic illustration of the mechanisms considered in the present study along with the highest energy barrier along each respective pathway. (a) Capture of conduction band electrons at electron traps. (b) Landscape crossover by thermal excitation. (c) Nonradiative recombination via oxygen vacancies.

much faster than the thermal crossing of the landscapes rate in dilute YAG:Ce at temperatures above $573 \mathrm{~K}^{2}$

While a single mechanism can dominate under certain conditions, such as high Ce content or low temperatures, luminescence quenching is likely due to a combination of the abovementioned processes. ${ }^{6}$ It is, however, difficult to discriminate their contributions based on experimental analysis alone. This pertains in particular to mechanisms (ii) and (iii), which involve electron traps and/or killer centers provided by defects, the properties of which are notoriously difficult to access experimentally. ${ }^{2,11}$

Here, using first-principles calculations, we analyze the mechanisms described above and identify a detailed recombination pathway involving oxygen vacancies. While our calculations are specific for YAG:Ce, the key parameters of the proposed mechanism (deep transition levels and strong localized electron-phonon coupling) are generic and can be found in many other wide-band gap materials, including oxides. ${ }^{12}$ Our results thereby point to specific mechanisms by which defects contribute to nonradiative recombination processes. This insight is relevant for a broad class of materials and enables more directed approaches to mediating and controlling nonradiative decay processes. As part of our analysis of luminescence quenching, we also probe the coupling between optical transitions and vibrational degrees of freedom using the generating function approach. ${ }^{13} \mathrm{We}$ demonstrate that the phonon sidebands in Ce absorption and emission spectra, which have been previously associated with localized defect modes, ${ }^{1,14}$ are in fact due to a superposition of many delocalized modes, an important finding in the context of the thermally activated landscape crossover model.

\section{METHODOLOGY}

2.1. Lineshapes. Optical lineshapes are modeled using the generating function approach ${ }^{13,15,16}$ under the FranckCondon, parallel mode, and low-temperature approximations, which results in a computationally feasible scheme. ${ }^{17}$ To begin with, we define the coordinate $Q_{\nu}$ as

$$
Q_{\nu}=\sum_{a} \sqrt{m_{a}}\left(\boldsymbol{R}_{a}^{i}-\boldsymbol{R}_{a}{ }^{f}\right) \cdot u_{\nu, a}
$$

where $u_{\nu}$ is the normalized displacement vector of the phonon branch $\nu$. The partial Huang-Rhys (HR) factor is defined as $S_{\nu}=1 / 2 \omega_{k} Q_{\nu}{ }^{2}$. In the parallel mode approximation, the normal modes of the initial and final states, and hence $Q_{\nu}$, are assumed to be related by a simple translation.

The lineshape function for absorption is

$$
A\left(\omega_{\mathrm{eg}}-\omega\right)=\frac{1}{2 \pi} \int_{-\infty}^{\infty} e^{i \omega t-\kappa t} G(t) \mathrm{d} t
$$

where $\kappa$ determines the lifetime broadening of individual contributions, $\omega_{\mathrm{eg}}$ is the angular frequency corresponding to the transition between the ground and excited states, and the generating function $G(t)$ is given by

$$
G(t)=\exp \left[S(t)-\sum_{\nu} S_{\nu}\right]
$$

Here, $S(t)$ is the Fourier transform of the spectral function

$$
S(\omega)=\sum_{\nu} S_{\nu} \delta\left(\omega-\omega_{\nu}\right)
$$

where the $\delta$-functions in practice are approximated with normalized Gaussian functions. The emission intensity is proportional to $\omega^{3} A(\omega)$, and the excitation intensity is proportional to $\omega A(\omega) .^{18}$

2.2. Computational Details. Electronic structure calculations were performed within the framework of density functional theory (DFT) using the projector-augmented wave method, ${ }^{19,20}$ as implemented in the Vienna ab initio simulation package $^{21,22}$ with a plane wave cutoff energy of $500 \mathrm{eV}$. The exchange-correlation potential was approximated with the PBE functional. ${ }^{23}$ Throughout this work, the DFT $+U$ method $^{24}$ was used with $U=2.5 \mathrm{eV}$ applied to $\mathrm{Ce} 4 \mathrm{f}$ states, which has been shown to yield accurate positions of the $\mathrm{Ce} 4 \mathrm{f}$ states in a range of phosphors. ${ }^{25}$ The calculations on $\mathrm{Ce}$ defects in the excited state were performed by constraining the occupations of $4 \mathrm{f}$ and $5 \mathrm{~d}$ states. Spin-orbit coupling was included in the emission spectrum by the addition of an extra peak shifted by $0.35 \mathrm{eV}$ and redistribution of the spectral weight. The shift was extracted from the energy difference of the ${ }^{2} F_{5 / 2}$ and ${ }^{2} F_{7 / 2}$ states in ref 26 , while the intensity ratio is given by the multiplicities.

Most defect calculations were carried out using the 160atom conventional (simple cubic) unit cell, while the Brillouin zone was sampled using a zone centered $2 \times 2 \times 2$ k-point mesh. The ionic positions were allowed to relax until all forces fell below $20 \mathrm{meV} / \AA$. Phonon and lineshape calculations were performed using a 320-atom supercell, and the Brillouin zone was sampled at the zone center only. An extended KrögerVink notation is used where $\mathrm{Ce}_{\mathrm{Y}}^{\times}$is a short notation for $\mathrm{Ce}$ $\left(4 \mathrm{f}^{1} 5 \mathrm{~d}^{0}\right), \mathrm{Ce}_{\mathrm{Y}}^{*}$ for $\mathrm{Ce}\left(4 \mathrm{f}^{0} 5 \mathrm{~d}^{1}\right)$, and $\mathrm{Ce}_{\mathrm{Y}}^{\bullet}$ for Ce $\left(4 \mathrm{f}^{0} 5 \mathrm{~d}^{0}\right)$.

2.3. Semilocal vs Hybrid Functionals. The $\mathrm{PBE}$ functional yields a band gap of $4.6 \mathrm{eV}$, which, as is common for semi-local functionals, is significantly smaller than 
experimental values that are found in the range of $6.4^{27}$ to 7.7 $\mathrm{eV}$, as discussed in ref 26 . The band gap underestimation can be compensated using hybrid functionals such as PBE0, ${ }^{29}$ which using a mixing parameter of 0.32 yields a band gap of 7.8 $\mathrm{eV}$, in much better agreement with experimental data. When going from $\mathrm{PBE}$ to $\mathrm{PBE} 0$, valence band maximum and conduction band minimum (CBM) shift by 2.1 and $1.1 \mathrm{eV}$, respectively (see Figure S4). ${ }^{28}$

For the oxygen vacancies, we therefore also carried out PBE0 calculations, for which we employed 80-atom cells and zone center Brillouin zone sampling. Similar calculations for Ce-doped systems were, however, found to yield erroneous results for the excited state. In these calculations, the $4 \mathrm{f}-5 \mathrm{~d}$ gap strongly (and nonphysically) decreases with increasing mixing parameter, leading to a level crossover in the $\mathrm{Ce}_{Y}^{\times}$ configuration already for mixing parameters below 0.32 . We attribute this behavior to the inability of the PBE0 functional (as well as other common hybrid functionals) to account for differences in the screening of $4 \mathrm{f}$ and $5 \mathrm{~d}$ states, as already noted in earlier studies on $\operatorname{LaBr} 3_{3} .^{30,31}$ In all calculations involving Ce reported below, we therefore used exclusively the DFT $+U$ method. Where necessary (Section 3.3), band gap errors were compensated by using the PBE $\rightarrow$ PBE0 band edge shifts reported above, as described in ref 32 .

\section{RESULTS}

3.1. Ground and Excited-State Landscapes. YAG crystallizes in space group $\mathrm{I} a \overline{3} d$ (ITC number 230) with atoms on Wyckoff sites $24 c(\mathrm{Y}), 16 a / 24 d(\mathrm{Al})$, and $96 h(\mathrm{O})$. The calculated lattice parameter is $12.108 \AA$ compared with an experimental value of $12.01 \pm 0.02 \AA^{33}$

In the ground state $\left(\mathrm{Ce}: 4 \mathrm{f}^{1} 5 \mathrm{~d}^{0}\right)$, the substitution of $\mathrm{Ce}$ on a $\mathrm{Y}$ site causes an outward relaxation of the nearest oxygen sites by $0.07 \AA$ on average. If the $4 \mathrm{f}$ electron is promoted to a $5 \mathrm{~d}$ state, it becomes more delocalized, which effectively reduces the size of the Ce ion and causes an inward relaxation of the oxygen neighbors by $0.06 \AA$ on average, such that the local geometry is almost identical to the Ce-free lattice. The configuration coordinate (CC) diagram is then obtained by linear interpolation between the fully relaxed ground and excited state configurations (Figure 2). From the total energies, we obtain an absorption energy of $2.67 \mathrm{eV}$ and an emission energy of $2.21 \mathrm{eV}$, which are in very good agreement with experimental values of 2.70 and $2.31 \mathrm{eV}$, respectively. ${ }^{1}$ The Stokes shifts are 0.25 and $0.22 \mathrm{eV}$ for emission and absorption, respectively, again in good agreement with the experimental value of $0.30 \mathrm{eV} .^{1}$ Finally, our results are in agreement with earlier calculations of the absorption and emission energies with small quantitative differences due to different computational parameters. ${ }^{34}$

We note that in this case, the energy differences between the 4f and 5d Kohn-Sham levels do not even semi-quantitatively correspond to the transition energies obtained from the total energies (see Figure S1). This highlights the fact that while the Kohn-Sham levels can often be helpful starting points for quasi-particle descriptions, in general, they cannot be interpreted as quasi-particle energies themselves.

A rough estimate for the crossing point between ground and excited-state landscapes can be obtained by fitting a quadratic polynomial to the energies in the vicinity of the respective equilibrium geometries. This yields a barrier of $3.75 \mathrm{eV}$. While this is a rough approximation of the upper limit, this very high energy barrier nonetheless strongly suggests that thermally
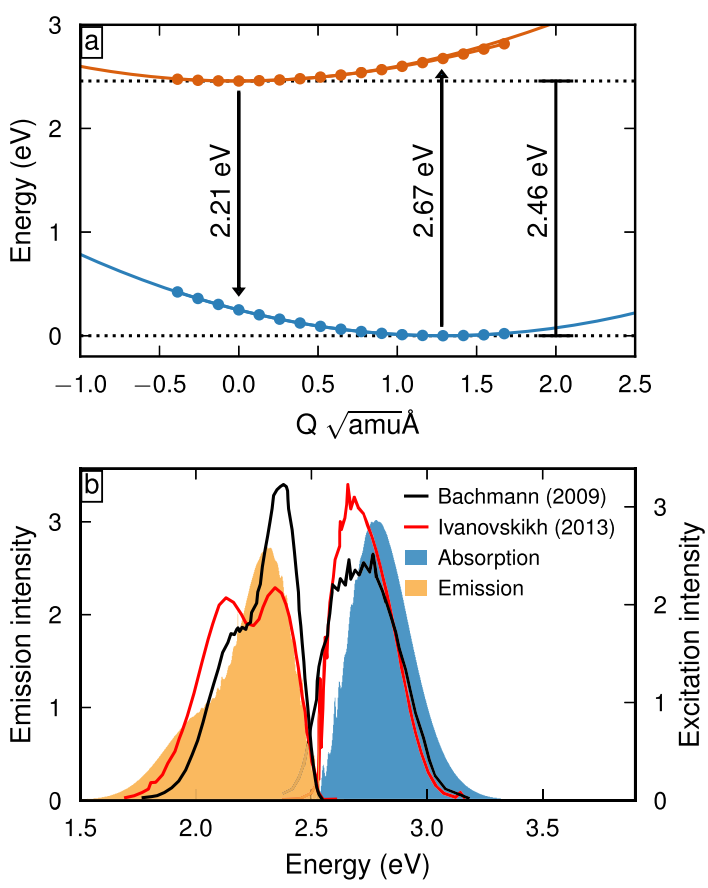

Figure 2. (a) 1D configuration coordinate diagram for the $4 f-5 d$ transition. Solid lines are quadratic fits to the potential energies. (b) Normalized optical lineshapes as computed with the generating function method. Black lines are experimental measurements from ref 1 performed at $5 \mathrm{~K}$ for the emission spectrum and at $300 \mathrm{~K}$ for the absorption spectrum. Red lines are low-temperature optical spectra from ref 26. The computed optical lineshapes have been shifted to the experimental ZPLs of ref 1 to simplify comparison.

activated crossing of landscapes is unlikely to be an important factor, in agreement with earlier assessments. ${ }^{10}$ The estimated energy barrier is an upper limit, and it is likely that the energy barrier will be smaller in more sophisticated models that go beyond the one-dimensional configurational coordinate model.

3.2. Vibrational Coupling to the $4 f-5 d$ Transition. The vibrational broadening of absorption and emission spectra is an important feature for the functionality of any material used for photon downconversion. It also provides important information concerning the energy landscape connecting the ground and excitedstate geometries. As a result, a lot of spectroscopic data are available for YAG:Ce, which provides an opportunity to validate our calculations in more detail and to gain further insight into the energy landscape.

Our calculated spectra are in good overall agreement with experimental data ${ }^{1,26}$ (Figure 2b), and the Stokes shifts computed from the spectra as the difference between the absorption maximum at $2.71 \mathrm{eV}$ and the emission intensity maximum at $2.24 \mathrm{eV}$ are close to the values obtained from the CC diagram (Figure 2a).

The fine structure of the spectra in the vicinity of the zerophonon line $(\mathrm{ZPL})$ provides important information concerning the phonon modes that couple to the electronic transitions and the CC diagram such as the total HR factor. Our calculations successfully reproduce the experimentally observed features in the fine structure of the optical spectra ${ }^{1,14}$ also in this regard (Figure 3a), including the total HR factor, for which we obtain 5.5 using the normal modes for the ground state, compared with an experimental value of $6 .^{1}$

Based on the experimental measurements, ${ }^{1,14}$ it has been proposed that the fine structure of the optical spectra 
originates from a single mode around $25 \mathrm{meV}$ (or $200 \mathrm{~cm}^{-1}$ ) with higher energy features being replicas of this mode. The presence of such a, presumably localized, mode would then also largely coincide with the CC. We can query the validity of this interpretation by analyzing the spectral function $S(\omega)$, which underlies the lineshape calculation (Section 2.1) and whose structure is reflected in the optical spectra. This analysis reveals that the fine structure of the optical spectra originates from coupling to a large number of modes (Figure $3 \mathrm{~b}$ ) in contrast to the earlier interpretations alluded to above. ${ }^{1,14}$

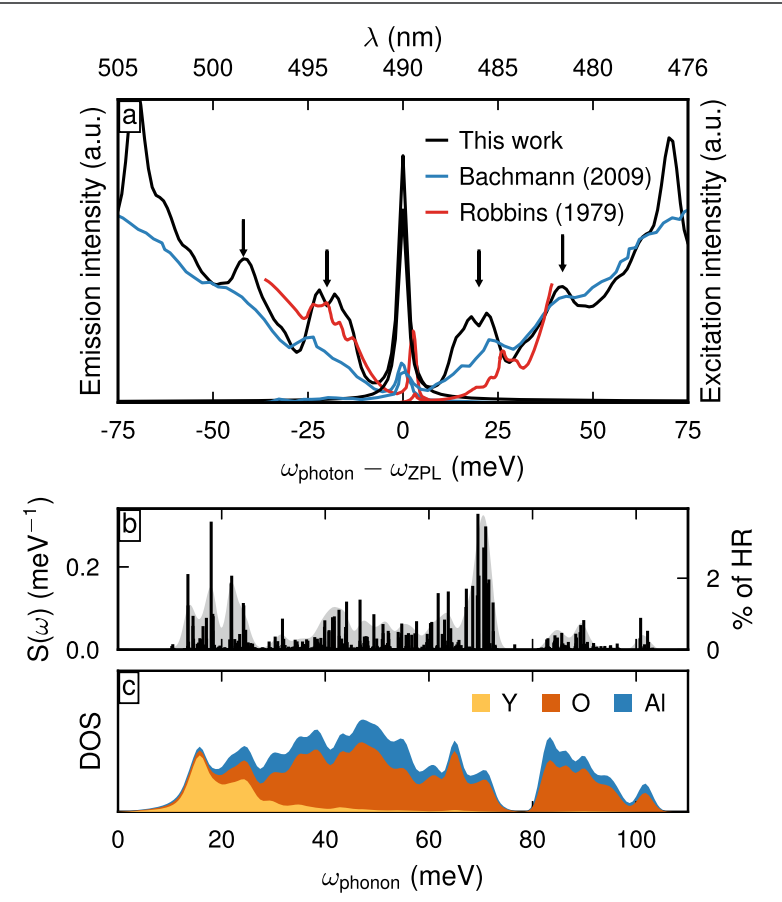

Figure 3. (a) Fine structure of the lineshape around the ZPL. Experimental data are taken from ref 1 [Bachmann (2009)] and ref 14 [Robbins (1979)]. Experimental intensities were scaled to match the intensity of the computed spectra. The ZPL in the computed results has been shifted to the experimental value of ref 1. (b) Electronphonon spectral function (shaded) and its corresponding partial HR factors. (c) Phonon density of states decomposed into contributions from different atomic species.

The main features are two distinct bands at approximately 20 and $70 \mathrm{meV}$ and a less pronounced band around $40 \mathrm{meV}$. The $20 \mathrm{meV}$ band is associated with dispersive yttriumdominated modes, while the $70 \mathrm{meV}$ band is due to dispersive oxygen-dominated modes, including motion of the nearestneighbor oxygen atoms of $\mathrm{Ce}$. At $20 \mathrm{meV}$, the phonon density of states is dominated by modes associated with $\mathrm{Y}$ motion (Figure 3c). Importantly, there are, however, no significant contributions from localized (defect) modes and there is no mode that contributes more than $4 \%$ to the total HR factor. The calculations thus demonstrate that the fine structure of the optical spectra can be entirely explained by delocalized modes and that there is no distinct localized mode that can account for relaxation along the CC.

3.3. Ionization of the $5 d$ State. Having addressed the character of ground and excited landscapes and their approximate connection to thermally activated landscape crossover (mechanism i), we now address the thermal ionization model (mechanism iii). According to the latter, transitions from the excited $5 \mathrm{~d}$ state to the conduction band manifold would result in luminescence quenching if the electron is subsequently captured at another site.

To evaluate the activation energy for ionization of the $5 \mathrm{~d}$ state, we require its position with respect to the conduction band edge. As noted above (Section 3.1), the Kohn-Sham eigenvalues do, however, not provide sensible guidance in the present case. We therefore again resort to total energy differences instead and more precisely the relative defect formation energies (Figure 4), as the ionization barrier

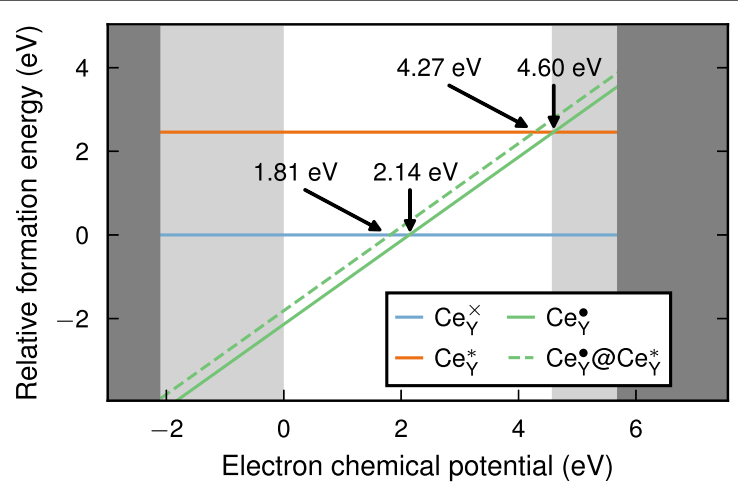

Figure 4. Relative formation energy of the $\mathrm{Ce}_{\mathrm{Y}}$ substitutional defect as a function of electron chemical potential. The PBE0 band edge shifts have been superimposed on the PBE values.

correspond to the charge transition level (CTL) from $\mathrm{Ce}_{\mathrm{Y}}^{\bullet}$ to $\mathrm{Ce}_{Y}^{*}$. Taking into account the band edge shift from PBE to PBE0, this yields a value of $1.42 \mathrm{eV}$, when the $\mathrm{Ce}_{\mathrm{Y}}^{\bullet}$ state is fixed at the $\mathrm{Ce}_{Y}^{*}$ ionic configuration, corresponding to a vertical transition or photoionization. If the relaxation of $\mathrm{Ce}_{\mathrm{Y}}^{\bullet}$ is taken into account, CTL moves $0.33 \mathrm{eV}$ closer to the CBM, resulting in a transition energy of $1.08 \mathrm{eV}$. These values are in good agreement with estimations of the 5d-CBM distance of $0.76-$ $1.24 \mathrm{eV}^{2,35}$ The ionization energy is likely to be reduced at higher temperatures because of renormalization of the conduction band edge. This would place our prediction closer to the lower experimental value.

3.4. Charge Transfer to an Oxygen Vacancy. In many oxides, including $\mathrm{YAG}^{36,37}$ oxygen vacancies $\left(\mathrm{V}_{\mathrm{O}}\right)$ are among the most important intrinsic defects. ${ }^{12,38-40}$ In wide band gap oxides, oxygen vacancies commonly feature deep CTLs and electronic levels and exhibit substantial structural relaxation between different charge states. ${ }^{12}$ These defects could thus play important roles in nonradiative recombination processes. Here, we therefore consider in detail the charge transfer reaction from $\mathrm{Ce}$ to $\mathrm{V}_{\mathrm{O}}$ (Figure 5a) and assess the ability of oxygen vacancies to act as electron traps in the ionization mechanism (Figure 5b). We emphasize that even if oxygen vacancies are predominantly neutral for Fermi levels in the upper half of the band gap (the expected outcome after synthesis), positively charged oxygen vacancies are still the most probable donor defects by comparison with other candidates such as interstitials and antisites (also see Figure S3). They are thus required to maintain charge neutrality (balancing free electrons at the conduction band edge) and are electrically active. In the following, we therefore consider oxygen vacancies in charge states $+2,+1$, and 0 . (Negatively charged oxygen vacancies dissociate into a neutral vacancy and a free electron, see Figure S9).

The localized defect level associated with $\mathrm{V}_{\mathrm{O}}^{\times}$lies inside the band gap, and the associated charge density is localized in real 
a)

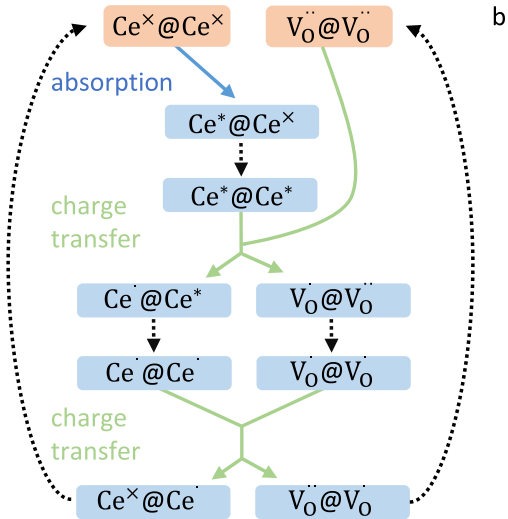

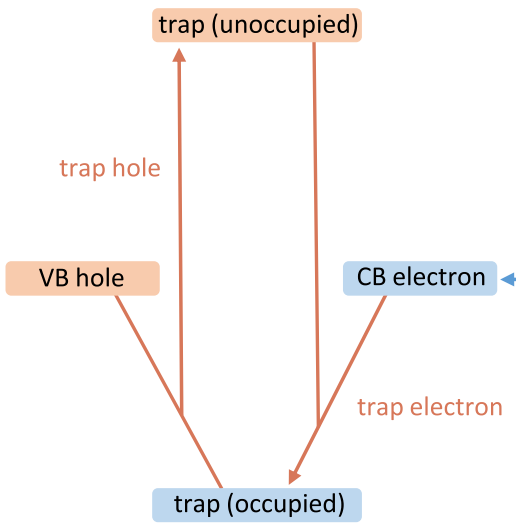

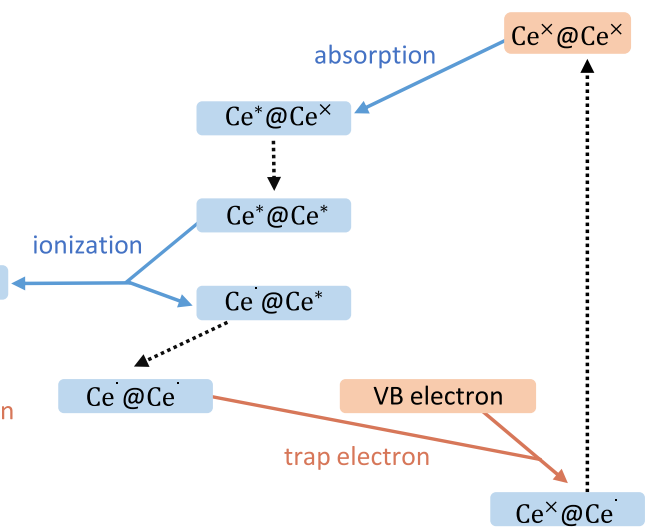

Figure 5. Illustration of reaction paths. (a) Thermal quenching via charge transfer to an oxygen vacancy. (b) Thermal quenching via electron transfer from the Ce $5 \mathrm{~d}$ state to the conduction band with subsequent trapping at a killer center.

space and has predominantly $s$-character, while the $+2 / 0 \mathrm{CTL}$ resides $1.79 \mathrm{eV}$ below the $\mathrm{CBM}$ at the $\mathrm{PBE}$ level and $3.00 \mathrm{eV}$ at the PBE0 level (see Supporting Information for the formation energy as a function of the electron chemical potential). These observations are consistent with the oxygen vacancy inducing a deep defect state. ${ }^{12}$ We note that the position of the $+2 / 0 \mathrm{CTL}$ is too deep to be identified as the electron traps experimentally found between $0.86 \mathrm{eV}$ and $1.52 \mathrm{eV}^{2}$ In $\mathrm{Lu}_{3} \mathrm{Al}_{5} \mathrm{O}_{12}$ (LuAG), it was found that antisites can act as shallow electron traps. ${ }^{41}$ In YAG, however, the antisites do not exhibit CTLs in the band gap (see Supporting Information). Even so, the $\mathrm{Al}_{\mathrm{Y}}{ }^{\prime}$ defect exhibits a localized Kohn-Sham state $0.3 \mathrm{eV}$ below the conduction band edge, which can indicate that it can act as a local electron acceptor.

As a result of its electronic structure, the oxygen vacancy can participate in a charge transfer mechanism with Ce (Figure 6). Immediately after absorption at a $\mathrm{Ce}$ site, the system undergoes a fast relaxation to the equilibrium geometry $\mathrm{Ce}_{\mathrm{Y}}^{*}$, dissipating $0.22 \mathrm{eV}$ in the process (Figure $2 \mathrm{a}$ and relaxation along segment $\mathrm{I}$ as shown in Figure 6).

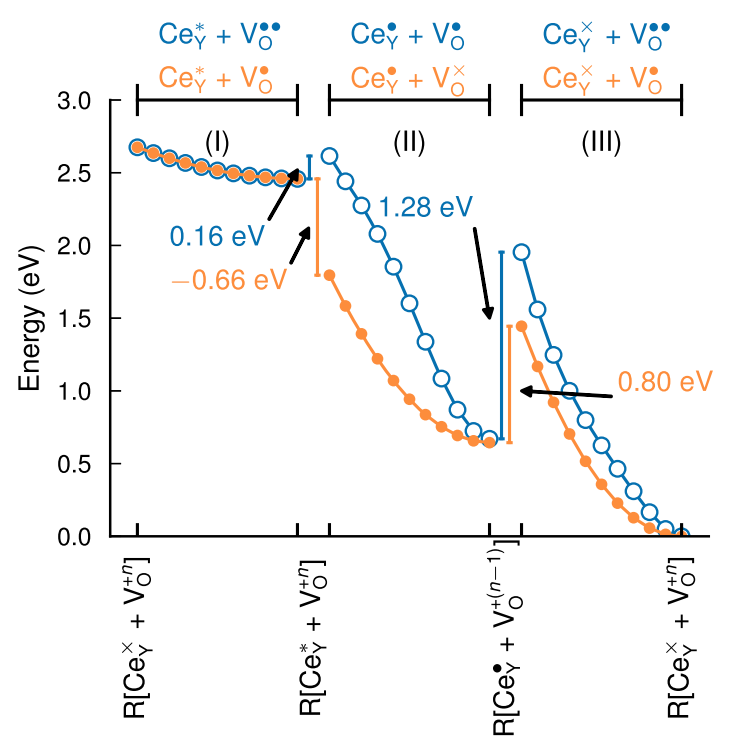

Figure 6. Thermal deactivation via oxygen vacancies. Points represent the sum of the total energy of $\mathrm{Ce}$ and $\mathrm{V}_{\mathrm{O}}$ defects for the same total charge states. The configurations on the abscissa refer to the equilibrium configuration of the respective combination of charge states.
At this point, the excitation can migrate through the system by resonant transfer between Ce sites. This process is practical if the absorption and emission spectra overlap, ${ }^{42}$ which is increasingly the case as the temperature goes up. ${ }^{1}$ Alternatively, transfer can occur by non-zero exchange interaction. This requires Ce sites to be sufficiently close to each other, which is the case if $\mathrm{Ce}$ concentrations reach the percent range. Resonant transfer can proceed until emission occurs or until the excitation reaches a $\mathrm{Ce}$ atom that is in spatial proximity to either a singly $\left(\mathrm{V}_{\mathrm{O}}^{\bullet}\right)$ or doubly charged oxygen vacancy $\left(\mathrm{V}_{\mathrm{O}}^{\bullet \bullet}\right)$. In practice, the Ce concentration can be assumed to be much larger than the oxygen vacancy concentration, making it plausible that a large fraction of oxygen vacancies have a Ce atom in their proximity (right-most point of segment $\mathrm{I}$ in Figure 6).

After reaching a $\mathrm{Ce}_{\mathrm{Y}}^{*}-\mathrm{V}_{\mathrm{O}}^{\bullet}$ or $\mathrm{Ce}_{\mathrm{Y}}^{*}-\mathrm{V}_{\mathrm{O}}^{\bullet \bullet}$ configuration, the electron in the $\mathrm{Ce}_{\mathrm{Y}}^{*}$ state can be transferred from the Ce atom to the oxygen vacancy (transition from segment I to segment II, as shown in Figure 6), leading to a charged Ce species $\left(\mathrm{Ce}_{\mathrm{Y}}^{\bullet}\right)$ and a neutral $\left(\mathrm{V}_{\mathrm{O}}^{\times}\right)$or singly charged vacancy $\left(\mathrm{V}_{\mathrm{O}}^{\bullet}\right)$. The former transition leads to a reduction in the total energy by $0.66 \mathrm{eV}$, whereas the latter is associated with a small energy increase of $0.16 \mathrm{eV}$.

The subsequent relaxation decreases the energy by $1.15 \mathrm{eV}$ $\left(\mathrm{Ce}_{\mathrm{Y}}^{\bullet}-\mathrm{V}_{\mathrm{O}}^{\times}\right)$and $1.94 \mathrm{eV}\left(\mathrm{Ce}_{\mathrm{Y}}^{\times}-\mathrm{V}_{\mathrm{O}}^{\bullet}\right)$, which needs to be dissipated via coupling to lattice vibrations, leading to local heating (segment II in Figure 6). The majority of this energy gain arises from the relaxation of the oxygen vacancy, while only $0.33 \mathrm{eV}$ is associated with the relaxation of the Ce site. The large relaxation energy implies that the system is effectively trapped in a low energy state. Already at this point, luminescence quenching has been achieved irreversibly. The lowest energy pathway to escape from this configuration leads back to $\mathrm{Ce}$ in its ground state configuration $\mathrm{Ce}_{\mathrm{Y}}^{\times}$in combination with either a singly or doubly charge oxygen vacancy with energy barriers of 0.80 and $1.28 \mathrm{eV}$, respectively (transition from segment I to segment II, as shown in Figure 6).

\section{DISCUSSION}

In the preceding sections, using first-principles calculations, we obtained the limiting energy barriers for different recombination pathways. Specifically, we obtained an approximate upper barrier of $3.75 \mathrm{eV}$ for the thermally activated landscape crossover (mechanism i; Section 3.1) and a barrier of $1.08 \mathrm{eV}$ 
for the thermal ionization from occupied Ce $5 \mathrm{~d}$ states (mechanism iii; Section 3.3). In addition, we established a specific nonradiative recombination pathway in conjunction with resonant excitation transfer (mechanism ii) that involves oxygen vacancies (Section 3.4). In the latter case, we found the maximum barriers along the reaction pathway to be $0.80 \mathrm{eV}$ for charge transfer via $\mathrm{V}_{\mathrm{O}}^{\bullet}$ and $1.28 \mathrm{eV}$ for charge transfer via $\mathrm{V}_{\mathrm{O}}^{\bullet \bullet}$. The charge transfer mechanism via oxygen vacancies thus yields by far the lowest activation barrier among the mechanisms considered here (Figure 1).

The energy barriers are on their own insufficient to quantitatively predict the rates for these recombination mechanisms. They are, however, usually the most important parameter; for most mechanisms, transition rates are exponentially dependent on the activation energy. ${ }^{43}$ The charge transfer mechanism proposed here should thus be an important contribution to the nonradiative recombination in Ce-doped YAG.

Oxygen vacancies in YAG exhibit very strong and localized electron-phonon coupling. The latter can be attributed to pronounced charge localization and the freedom for the atoms surrounding the vacancy to relax. This allows oxygen vacancies to dissipate large amounts of energy locally while acting as a local acceptor relative to $\mathrm{Ce}_{\mathrm{Y}}^{\times}$. The limiting step in this mechanism is the transition from $\mathrm{Ce}_{\mathrm{Y}}^{\bullet}-\mathrm{V}_{\mathrm{O}}^{\times}$to $\mathrm{Ce}_{\mathrm{Y}}^{\times}-\mathrm{V}_{\mathrm{O}}^{\bullet}$, at which point $\mathrm{Ce}$ is oxidized and therefore optically inactive.

In support of the charge transfer mechanism, it has been shown by an $\mathrm{X}$-ray absorption near-edge structure that $\mathrm{Ce}_{\mathrm{Y}}^{\bullet}$ can coexist with $\mathrm{Ce}_{\mathrm{Y}}^{\times}$in $\mathrm{YAG}: \mathrm{Ce}^{44}$ and that the presence of electron acceptors in the vicinity of $\mathrm{Ce}$ can oxidize the $\mathrm{Ce}$ atom in related materials. ${ }^{45}$ One might suspect transition metal impurities to participate in a similar reaction. The screening from the transition metal valence states and the much more restrictive geometry make it, however, unlikely that they dissipate similarly larger quantities of energy via a charge transfer mechanism.

The involvement of oxygen vacancies in the recombination process in Ce-doped oxide phosphors has been considered before. In ref 46, the authors concluded that landscape crossover and $5 \mathrm{~d}$ ionization could not satisfactorily explain the luminescence quenching in $\mathrm{Lu}_{2} \mathrm{SiO}_{5}$ : Ce. A hole autoionization process was therefore proposed in which $\mathrm{V}_{\mathrm{O}}^{\times}$reduced the $\mathrm{Ce}$ atom, leading to a $4 \mathrm{f}^{1} 5 \mathrm{f}^{1}$ electronic configuration. While this model is conceptually different from the idea of oxygen vacancies as local energy dissipators presented here, it shares some features. The hole autoionization model of ref 46 involves reduction from $\mathrm{Ce}_{\mathrm{Y}}^{\times}$to $\mathrm{Ce}_{\mathrm{Y}}{ }^{\prime}$ and oxidation of the oxygen vacancy, while in the model proposed here, the charge transfer in the first step of the reaction occurs in the opposite direction.

Finally, we note that the mechanism proposed in this study relies on general characteristics of oxygen vacancies in widegap oxides, most importantly their deep character, which entails strong relaxation between charge states and localized charges. ${ }^{12}$ One can therefore anticipate it to be applicable also in other oxide-based phosphors. Moreover, the oxygen vacancy concentration can, in principle, be controlled via the chemical environment during growth and annealing. This knob could be used to further assess the role of oxygen vacancies in luminescence quenching.

\section{CONCLUSIONS}

To summarize, we have provided energy estimates for the most plausible luminescence quenching mechanisms in YAG:Ce by first-principles calculations. The lowest energy pathway is obtained for a thermally activated concentration quenching mechanism that involves charge transfer between Ce atom and oxygen vacancies. In this pathway, the limiting energy barrier is comparable to the thermal ionization energy. As part of this investigation, we also analyzed the vibrational broadening and fine structure of the $4 \mathrm{f}-5 \mathrm{~d}$ transition on Ce. This analysis revealed that the fine structure of the phonon sidebands does not arise from a specific localized (defect) mode but is rather the result of a combination of many delocalized modes.

\section{ASSOCIATED CONTENT}

\section{Supporting Information}

The Supporting Information is available free of charge at https://pubs.acs.org/doi/10.1021/acs.chemmater.0c02449.

Kohn-Sham states for $\mathrm{Ce}_{\mathrm{Y}}$ and $\mathrm{V}_{\mathrm{O}}$ in different charge states, charge density corresponding to the localized level in $\mathrm{V}_{\mathrm{O}}^{\times}$, the relative formation energies of $\mathrm{V}_{\mathrm{O}}$ and antisite defects in different charge states, one-dimensional $\mathrm{CC}$ diagrams for $\mathrm{V}_{\mathrm{O}}$, finite-size effects on formation energies and spectral distribution functions, and the change in the displacements between $\mathrm{Ce}_{\mathrm{Y}}^{\times}$and $\mathrm{Ce}_{\mathrm{Y}}^{*}(\mathrm{PDF})$

\section{AUTHOR INFORMATION}

\section{Corresponding Author}

Paul Erhart - Department of Physics, Chalmers University of Technology, Gothenburg 41296, Sweden; 이이이.org/00000002-2516-6061; Email: erhart@chalmers.se

\section{Authors}

Christopher Linderälv - Department of Physics, Chalmers University of Technology, Gothenburg 41296, Sweden

Daniel Åberg - Physical and Life Sciences Directorate, Lawrence Livermore National Laboratory, Livermore 94550, California, United States

Complete contact information is available at:

https://pubs.acs.org/10.1021/acs.chemmater.0c02449

\section{Notes}

The authors declare no competing financial interest.

\section{ACKNOWLEDGMENTS}

We thank Maths Karlsson, Yuan-Chih Lin, and Magnus Engholm for fruitful discussions. Funding from the Knut and Alice Wallenberg Foundation (2014.0226) as well as the Swedish Research Council (2018-06482) is gratefully acknowledged. The computations were enabled by resources provided by the Swedish National Infrastructure for Computing (SNIC) at NSC, C3SE, and PDC partially funded by the Swedish Research Council through grant agreement no. 2018-05973. Part of this work was performed under the auspices of the U.S. Department of Energy by Lawrence Livermore National Laboratory under contract DE-AC52-07NA27344 with support from the National Nuclear Security Administration Office of Nonproliferation Research and Development (NA-22). 


\section{REFERENCES}

(1) Bachmann, V.; Ronda, C.; Meijerink, A. Temperature Quenching of Yellow $\mathrm{Ce}^{3+}$ Luminescence in YAG:Ce. Chem. Mater. 2009, 21, 2077-2084.

(2) Ueda, J.; Dorenbos, P.; Bos, A. J. J.; Meijerink, A.; Tanabe, S. Insight into the Thermal Quenching Mechanism for $\mathrm{Y}_{3} \mathrm{Al}_{5} \mathrm{O}_{12}: \mathrm{Ce}^{3+}$ through Thermoluminescence Excitation Spectroscopy. J. Phys. Chem. C 2015, 119, 25003-25008.

(3) Nishiura, S.; Tanabe, S.; Fujioka, K.; Fujimoto, Y. Properties of transparent Ce:YAG ceramic phosphors for white LED. Opt. Mater. 2011, 33, 688.

(4) Lin, Y.-C.; Karlsson, M.; Bettinelli, M. Inorganic Phosphor Materials for Lighting. Top. Curr. Chem. 2016, 374, 21.

(5) Lin, Y.-C.; Erhart, P.; Bettinelli, M.; George, N. C.; Parker, S. F.; Karlsson, M. Understanding the Interactions between Vibrational Modes and Excited State Relaxation in $\mathrm{Y}_{3-x} \mathrm{Ce}_{x} \mathrm{Al}_{5} \mathrm{O}_{12}$ : Design Principles for Phosphors Based on $5 d-4 f$ Transitions. Chem. Mater. 2018, 30, 1865-1877.

(6) Lin, Y.-C.; Bettinelli, M.; Sharma, S. K.; Redlich, B.; Speghini, A.; Karlsson, M. Unraveling the impact of different thermal quenching routes on the luminescence efficiency of the $\mathrm{Y}_{3} \mathrm{Al}_{5} \mathrm{O}_{12}: \mathrm{Ce}^{3+}$ phosphor for white light emitting diodes. J. Mater. Chem. C 2020, 8, 1401514027.

(7) Bleijenberg, K. C.; Blasse, G. QMSCC calculations on thermal quenching of model phosphor systems. J. Solid State Chem. 1979, 28, 303-307.

(8) Blasse, G. Luminescence of inorganic solids: From isolated centres to concentrated systems. Prog. Solid State Chem. 1988, 18, $79-171$.

(9) George, N. C.; Pell, A. J.; Dantelle, G.; Page, K.; Llobet, A.; Balasubramanian, M.; Pintacuda, G.; Chmelka, B. F.; Seshadri, R. Local Environments of Dilute Activator Ions in the Solid-State Lighting Phosphor $\mathrm{Y}_{3-x} \mathrm{Ce}_{x} \mathrm{Al}_{5} \mathrm{O}_{12}$. Chem. Mater. 2013, 25, 39793995.

(10) Weber, M. J. Nonradiative decay from $5 d$ states of rare earths in crystals. Solid State Commun. 1973, 12, 741-744.

(11) Zych, E.; Brecher, C.; Glodo, J. Kinetics of Cerium Emission in a YAG:Ce Single Crystal: The Role of Traps. J. Phys.: Condens. Matter 2000, 12, 1947.

(12) Linderälv, C.; Lindman, A.; Erhart, P. A Unifying Perspective on Oxygen Vacancies in Wide Band Gap Oxides. J. Phys. Chem. Lett. 2018, 9, 222.

(13) Markham, J. J. Interaction of Normal Modes with Electron Traps. Rev. Mod. Phys. 1959, 31, 956-989.

(14) Robbins, D. J. The Effects of Crystal Field and Temperature on the Photoluminescence Excitation Efficiency of $\mathrm{Ce}^{3+}$ in YAG. $J$. Electrochem. Soc. 1979, 126, 1550-1555.

(15) Kubo, R.; Toyozawa, Y. Application of the Method of Generating Function to Radiative and Non-Radiative Transitions of a Trapped Electron in a Crystal. Prog. Theor. Phys. 1955, 13, 160182.

(16) Lax, M. The Franck-Condon Principle and Its Application to Crystals. J. Chem. Phys. 1952, 20, 1752-1760.

(17) Alkauskas, A.; Buckley, B. B.; Awschalom, D. D.; Van de Walle, C. G. First-principles theory of the luminescence lineshape for the triplet transition in diamond NV centres. New J. Phys. 2014, 16, 073026.

(18) Baiardi, A.; Bloino, J.; Barone, V. General Time Dependent Approach to Vibronic Spectroscopy Including Franck-Condon, Herzberg-Teller, and Duschinsky Effects. J. Chem. Theory Comput. 2013, 9, 4097-4115.

(19) Blöchl, P. E. Projector augmented-wave method. Phys. Rev. B 1994, 50, 17953-17979.

(20) Kresse, G.; Joubert, D. From ultrasoft pseudopotentials to the projector augmented-wave method. Phys. Rev. B: Condens. Matter Mater. Phys. 1999, 59, 1758-1775.

(21) Kresse, G.; Furthmüller, J. Efficiency of ab-initio total energy calculations for metals and semiconductors using a plane-wave basis set. Comput. Mater. Sci. 1996, 6, 15-50.
(22) Kresse, G.; Hafner, J. Ab initio molecular dynamics for liquid metals. Phys. Rev. B: Condens. Matter Mater. Phys. 1993, 47, 558-561.

(23) Perdew, J. P.; Burke, K.; Ernzerhof, M. Generalized Gradient Approximation Made Simple. Phys. Rev. Lett. 1996, 77, 3865-3868.

(24) Dudarev, S. L.; Botton, G. A.; Savrasov, S. Y.; Humphreys, C. J.; Sutton, A. P. Electron-energy-loss spectra and the structural stability of nickel oxide: An LSDA+U study. Phys. Rev. B: Condens. Matter Mater. Phys. 1998, 57, 1505-1509.

(25) Canning, A.; Chaudhry, A.; Boutchko, R.; Grønbech-Jensen, N. First-principles study of luminescence in Ce-doped inorganic scintillators. Phys. Rev. B: Condens. Matter Mater. Phys. 2011, 83, 125115.

(26) Ivanovskikh, K. V.; Ogiegło, J. M.; Zych, A.; Ronda, C. R.; Meijerink, A. Luminescence Temperature Quenching for $\mathrm{Ce}^{3+}$ and $\operatorname{Pr}^{3+} d-f$ Emission in YAG and LuAG. ECS J. Solid State Sci. Technol. 2013, 2, R3148-R3152.

(27) Slack, G. A.; Oliver, D. W.; Chrenko, R. M.; Roberts, S. Optical Absorption of $\mathrm{Y}_{3} \mathrm{Al}_{5} \mathrm{O}_{12}$ from 10 to $55,000 \mathrm{~cm}^{-1}$ Wave Numbers. Phys. Rev. 1969, 177, 1308-1314.

(28) Ning, L.; Ji, X.; Dong, Y.; Jin, W.; Huang, Y.; Pan, Z.; Tanner, P. A. First-principles study of Ce-doped $\mathrm{Y}_{3} \mathrm{Al}_{5} \mathrm{O}_{12}$ with $\mathrm{Si}-\mathrm{N}$ incorporation: electronic structures and optical properties. J. Mater. Chem. C 2016, 4, 5214-5221.

(29) Adamo, C.; Barone, V. Toward reliable density functional methods without adjustable parameters: The PBE0 model. J. Chem. Phys. 1999, 110, 6158.

(30) Åberg, D.; Sadigh, B.; Erhart, P. Electronic structure of $\mathrm{LaBr}_{3}$ from quasiparticle self-consistent $G W$ calculations. Phys. Rev. B: Condens. Matter Mater. Phys. 2012, 85, 125134.

(31) Erhart, P.; Sadigh, B.; Schleife, A.; Åberg, D. First-principles study of codoping in lanthanum bromide. Phys. Rev. B: Condens. Matter Mater. Phys. 2015, 91, 165206.

(32) Lindman, A.; Erhart, P.; Wahnström, G. Implications of the band gap problem on oxidation and hydration in acceptor-doped barium zirconate. Phys. Rev. B: Condens. Matter Mater. Phys. 2015, 91, 245114.

(33) Yoder, H. S.; Keith, M. L. Complete substitution of aluminum for silicon: The system $3 \mathrm{MnO} \cdot \mathrm{Al}_{2} \mathrm{O}_{3} \cdot 3 \mathrm{SiO}_{2}-3 \mathrm{Y}_{2} \mathrm{O}_{3} \cdot 5 \mathrm{Al}_{2} \mathrm{O}_{3} \cdot A m$. Mineral. 1951, 36, 519-533.

(34) Jia, Y.; Poncé, S.; Miglio, A.; Mikami, M.; Gonze, X. Assessment of First-Principles and Semiempirical Methodologies for Absorption and Emission Energies of $\mathrm{Ce}^{3+}$-Doped Luminescent Materials. Adv . Opt. Mater. 2017, 5, 1600997.

(35) Hamilton, D. S.; Gayen, S. K.; Pogatshnik, G. J.; Ghen, R. D.; Miniscalco, W. J. Optical-absorption and photoionization measurements from the excited states of $\mathrm{Ce}^{3+}: \mathrm{Y}_{3} \mathrm{Al}_{5} \mathrm{O}_{12}$. Phys. Rev. B: Condens. Matter Mater. Phys. 1989, 39, 8807-8815.

(36) Chen, J.; Lu, T. C.; Xu, Y.; Xu, A. G.; Chen, D. Q. Ab initio study of a charged vacancy in yttrium aluminum garnet $\left(\mathrm{Y}_{3} \mathrm{Al}_{5} \mathrm{O}_{12}\right)$. J. Phys.: Condens. Matter 2008, 20, 325212.

(37) Li, Z.; Liu, B.; Wang, J.; Sun, L.; Wang, J.; Zhou, Y. Mechanism of Intrinsic Point Defects and Oxygen Diffusion in Yttrium Aluminum Garnet: First-Principles Investigation. J. Am. Ceram. Soc. 2012, 95, $3628-3633$.

(38) Erhart, P. A first-principles study of helium storage in oxides and at oxide-iron interfaces. J. Appl. Phys. 2012, 111, 113502.

(39) Deml, A. M.; Holder, A. M.; O’Hayre, R. P.; Musgrave, C. B.; Stevanović, V. Intrinsic Material Properties Dictating Oxygen Vacancy Formation Energetics in Metal Oxides. J. Phys. Chem. Lett. 2015, 6, $1948-1953$.

(40) Nikl, M.; Laguta, V. V.; Vedda, A. Complex oxide scintillators: Material defects and scintillation performance. Phys. Status Solidi B 2008, 245, 1701-1722.

(41) Nikl, M.; Vedda, A.; Fasoli, M.; Fontana, I.; Laguta, V. V.; Mihokova, E.; Pejchal, J.; Rosa, J.; Nejezchleb, K. Shallow traps and radiative recombination processes in $\mathrm{Lu}_{3} \mathrm{Al}_{5} \mathrm{O}_{12}$ : Ce single crystal scintillator. Phys. Rev. B: Condens. Matter Mater. Phys. 2007, 76, 195121. 
(42) Blasse, G.; Grabmaier, B. C. Luminescent Materials; SpringerVerlag, 1994.

(43) Shi, L.; Xu, K.; Wang, L.-W. Comparative study of ab initio nonradiative recombination rate calculations under different formalisms. Phys. Rev. B: Condens. Matter Mater. Phys. 2015, 91, 205315.

(44) Dantelle, G.; Testemale, D.; Homeyer, E.; Cantarano, A.; Kodjikian, S.; Dujardin, C.; Hazemann, J.-L.; Ibanez, A. A new solvothermal method for the synthesis of size-controlled YAG:Ce single-nanocrystals. RSC Adv. 2018, 8, 26857-26870.

(45) Ueda, J.; Katayama, M.; Asami, K.; Xu, J.; Inada, Y.; Tanabe, S. Evidence of valence state change of $\mathrm{Ce}^{3+}$ and $\mathrm{Cr}^{3+}$ during UV charging process in $\mathrm{Y}_{3} \mathrm{Al}_{2} \mathrm{Ga}_{3} \mathrm{O}_{12}$ persistent phosphors. Opt. Mater. Express 2017, 7, 2471-2476.

(46) Jia, Y.; Miglio, A.; Mikami, M.; Gonze, X. Ab initio study of luminescence in Ce-doped $\mathrm{Lu}_{2} \mathrm{SiO}_{5}$ : The role of oxygen vacancies on emission color and thermal quenching behavior. Phys. Rev. Mater. 2018, 2, 125202. 